\title{
A Novel Approach for Analyzing the Electromagnetic Reflection from Stratified Anisotropic Media
}

\author{
Ming-Shing Lin, Ruey-Beei Wu, and Chun Hsiung Chen \\ Department of Electrical Engineering, \\ National Taiwan University, \\ Taipei, Taiwan, ROC
}

\begin{abstract}
This paper presents an analysis of plane-wave reflection from stratified anisotropic lossy media. To this end, an equivalent-transmission-line-circuit (ETLC) model is adopted in the frequency- and time-domain analysis. In this study, the reflection properties of graphite/epoxy, boron/epoxy, Kevlar/epoxy and their hybrid laminated composites are investigated in detail.
\end{abstract}

\section{INTRODUCTION}

The anisotropic materials such as microstrip substrate, radar absorbing materials, and laminated composites are widely employed in many engineering applications. The electromagnetic interaction with anisotropic materials thus deserves intensive investigation. Recently efforts by Morgan [1] and Titchener [2] employed the transition matrix method to study the reflection and transmission phenomena in anisotropic stratified media. The wave-transmission-matrix (WTM) method [3] was also used to solve the laminated and anisotropic composites illuminated by an obliquely incident plane wave. But, these methods cannot get the time-domain solution directly.

The laminated composites are increasingly used as replacement for metals in aircraft and aerospace applications due to their superior properties in strength-to-weight and modulus-toweight ratios. Therefore, the reflection problem associated with the anisotropic laminated composites needs special attention due to its close relation to the radar-cross-section problem. From the results of [2] and [3], it was suggested that one may use anisotropic media to reduce the reflection from metal structure.

In this paper, a novel approach based on the ETLC model is applied to analyze the reflection properties of various laminated composites. The major advantage of ETLC model [4] is that one may directly analyze the problem in both frequency and time domains.

\section{ETLC MODEL}

The ETLC model can handle the frequencyand time-domain propagation problem for inhomogeneous, anisotropic, and transversely lossy slab with permittivity $\left(\epsilon_{0} \overline{\bar{\epsilon}}\right)$, permeability $\left(\mu_{0} \overline{\bar{\mu}}\right)$, and conductivity $(\overline{\bar{\sigma}})$ tensors of the form

$\overline{\bar{\epsilon}}=\left[\begin{array}{ccc}\epsilon_{x x} & \epsilon_{x y} & 0 \\ \epsilon_{y x} & \epsilon_{y y} & 0 \\ 0 & 0 & \epsilon_{z z}\end{array}\right], \quad \overline{\bar{\mu}}=\left[\begin{array}{ccc}\mu_{x x} & \mu_{x y} & 0 \\ \mu_{y x} & \mu_{y y} & 0 \\ 0 & 0 & \epsilon_{z z}\end{array}\right]$ 


$$
\overline{\bar{\sigma}}=\left[\begin{array}{ccc}
\sigma_{x x} & \sigma_{x y} & 0 \\
\sigma_{y x} & \sigma_{y y} & 0 \\
0 & 0 & 0
\end{array}\right]
$$

This model is based on the analogy between field equations and coupled transmission line equations together with suitable boundary conditions. Thus, solving the electromagnetic propagation problem for the stratified anisotropic media is then equivalent to finding the frequency- and time-domain solutions from ETLC model. To facilitate the analysis, the plane wave is decom-

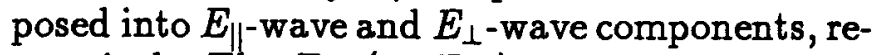
spectively. The $E_{\|^{-}}$(or $E_{\perp^{-}}$) wave means the one with E-field parallel (or perpendicular) to the $x-z$ plane of incidence.

Approximating the coupled transmission lines by cascading lumped T- and $\pi$ - circuits, one can solve the ETLC model by the circuit analysis programs such as PSPICE. From the output data of PSPICE program, we can get the reflection coefficients $R_{\|,\|}, R_{\|, \perp}, R_{\perp, \perp}$, and $R_{\perp, \|}$, in frequency domain, by the following definitions

$$
R_{p, q}=\frac{E_{p, q}^{r}}{E_{p}^{i}}
$$

Here the first subscript " $p$ " $=" \|$ " (or " $\perp$ ") denotes that the incident field $E^{i}$ is $E_{1-}$ (or $E_{\perp^{-}}$) wave, and the second subscript "q" = " " (or " $\perp$ ") denotes that the reflected field $E^{r}$ is $E_{\mid-}$(or $E_{\perp-}$ ) component.

In the time-domain analysis, the transient waveforms for reflected fields $\overline{\mathcal{E}}_{p, q}^{r}(t)$ may be obtained for any incident field waveform $\overline{\mathcal{E}}_{p}^{i}(\mathrm{t})$. The definitions of " $p$ " and " $q$ " are the same as those defined in the frequency-domain analysis.

\section{RESULTS AND DISCUSSIONS}

The wave reflection properties of anisotropic laminated composites backed by perfect electric conductor (PEC) (Fig.1) are then analyzed, based on a model which treats each lamina as a homogeneous and anisotropic sheet. In this study, the reflection properties of graphite/epoxy(G/E), boron/epoxy(B/E),
Kevlar/epoxy(K/E), and hybrid laminated composites backed by PEC are investigated in detail. Here, we regard the metal foil or metal coating as a PEC, which is usually used to enhance electromagnetic shielding. The parameters of these composite materials are listed in Table 1 . The parameters are described with respect to the composite's principal coordinates $\left(x^{\prime}, y^{\prime}, z^{\prime}\right)$. For theoretical analysis, the field quantities and the electrical parameters for each layer must be expressed in the global coordinates $(x, y, z)$, since composite layers with different principal axis directions are to be cascaded [3]. The numerical results are

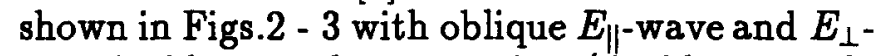
wave incident on the composites (incidence angle $\theta=0^{\circ}$ or $\left.45^{\circ}\right)$. In the computations of Figs.2 3 , each lamina has equal thickness and only the fiber orientation pattern $\left[0^{\circ} / 45^{\circ} / 90^{\circ} /-45^{\circ}\right]$ is considered.

Shown in Fig. 2 are the magnitudes of reflection coefficients for $G / E$ and $B / E$ laminated composites backed by PEC (laminate thickness $h=3 \times 10^{-3} \mathrm{~m}$ ) with $E_{\|-}$and $E_{\perp}$-wave incidence, respectively. For normal incidence case, the values of $\left|R_{\perp, \perp}\right|$ are smaller than those of $\left|R_{\|,\|}\right|$in most part of the frequency range. The values of $\left|R_{\|, \perp}\right|$ and $\left|R_{\perp, \|}\right|$ for $\mathrm{G} / \mathrm{E}$ composite are not shown because they are very small $\left(<10^{-3}\right)$. The values of $\left|R_{\|,\|,}\right|$and $\left|R_{\perp, \perp}\right|$ for $\mathrm{B} / \mathrm{E}$ are smaller than those for $\mathrm{G} / \mathrm{E}$ because the conductivity $\sigma_{x^{\prime}}$ of $\mathrm{B} / \mathrm{E}$ is much smaller than that of G/E. Interesting phenomenon observed is that there is a deep near $21 \mathrm{GHz}$ in the curves of $\left|R_{\perp, \perp}\right|$ for $\mathrm{B} / \mathrm{E}$.

Because the intrinsic properties of $\mathrm{K} / \mathrm{E}$ laminated composites are nearly isotropic, there is little difference in reflection properties between metal and $\mathrm{K} / \mathrm{E}$ laminated composites backed by PEC. Therefore, the results for $\mathrm{K} / \mathrm{E}$ laminated composites backed by PEC are not shown here.

The reflection properties of the hybrid composites with $B / E$ and G/E laminates backed by PEC are also calculated. It is noted that there is only a little difference in the reflection coefficients between this hybrid composites and the B/E composites backed by PEC. Therefore, the results are not shown here too. 
In the time-domain analysis, the Gaussian pulse defined by

$$
\mathcal{E}^{i}(t)=e^{-\left(\frac{t-t_{0}}{\tau}\right)^{2}} u(t),
$$

is chosen, where $\tau=5 \times 10^{-12} s, t_{0}=5 \times 10^{-11} s$, and

$$
u(t)= \begin{cases}1 & , t>0 \\ 0 & , t<0\end{cases}
$$

Four structures are studied here: (a) free space(FS) in regions $I$ and II, and G/E laminate in region III; (b) free space in regions I and II, and $B / E$ laminate in region III; (c) free space in region $\mathrm{I}, \mathrm{B} / \mathrm{E}$ laminate in region $\mathrm{II}$, and $\mathrm{G} / \mathrm{E}$ laminate in region III; and (d) free space in all regions. The structure and results are shown in Fig.3. From the delay time of reflected-wave peaks, one may estimate the reflection points within the structure. It is found that the reflection properties of the hybrid composites with $\mathrm{B} / \mathrm{E}$ and $\mathrm{G} / \mathrm{E}$ laminates are similar to those of $\mathrm{B} / \mathrm{E}$ laminated composite when the thickness $(h)$ of each region is $10^{-3} \mathrm{~m}$. It is also found that little wave energy reaches $\mathrm{PEC}$ in cases (a), (b), and (c), therefore, the reflection coefficients of laminated composites without PEC are the same as those with backed PEC. In other words, the reflection properties in cases (a), (b), and (c) are mainly governed by the composites, not by the backed PEC.

\section{CONCLUSIONS}

From the results obtained, some suggestion$s$ may also be made for reducing the reflection coefficients of laminated composites. First, B/E composite is a better selection for the outer laminated one. Second, the composites with $45^{\circ}$ lamina should be employed. Third, if metal coating is necessary for enhancing electromagnetic shielding, the coating should be applied on the backside of composites.

\section{ACKNOWLEDGMENT}

This work was supported by the National Science Council of the Republic of China under Grant NSC 82-0404-E-002-237.

\section{REFERENCES}

[1] M. A. Morgan, D. L. Fisher, and E. A. Milne, "Electromagnetic scattering by stratified inhomogeneous anisotropic media", IEEE Trans. on AP, Vol. AP-35, pp. 191-197, Feb. 1987.

[2] J. B. Titchener and J. R. Willis, "The reflection of electromagnetic waves from stratified anisotropic media," IEEE Trans. on AP, Vol. AP-39, pp. 35-39, Jan. 1991.

[3] M. S. Lin and C. H. Chen, "Plane-wave shielding properties of anisotropic laminated composites," IEEE Trans. on EMC., Vol. EMC-35, pp. 21-27, Feb. 1993.

[4] M. S. Lin, C. M. Lin, R. B. Wu, and C. H. Chen, "Transient Propagation in anisotropic laminated composites," IEEE Trans. Electromagn. Compat., Vol. EMC-35, Aug. 1993.

\section{AUTHORS}

Ming-Shing Lin, Senior engineer of Aeronautical Research Laboratory (ARL), Taichung, Taiwan. His current research interests include aircraft system electromagnetic compatibility, aircraft antennas, and electrical and electromagnetic properties of composites materials.

Ruey-Beei Wu, Professor of Electrical Engineering Department, National Taiwan University. He works primarily on the applications of numerical methods to electromagnetic field problems. He has been engaged in researches on dielectric waveguides, wave scattering of anisotropic objects, and interconnection modeling for computer packaging.

Chun Hsiung Chen, Professor of Electrical Engineering Department, National Taiwan University. His areas of interest include antenna and waveguide analysis, propagation and scattering of waves, and numerical techniques in electromagnetics. 
Fig.1. Geometry of M-ply laminated composites.

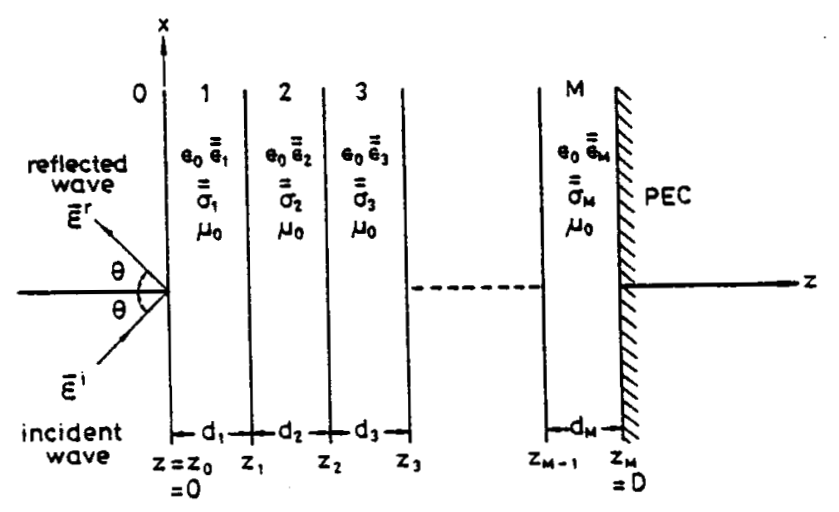

Fig.2. Reflectivity coefficients of 4-layer G/E and $\mathrm{B} / \mathrm{E}$ laminated composites backed by

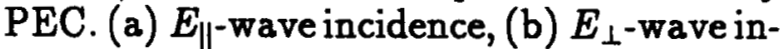
cidence.
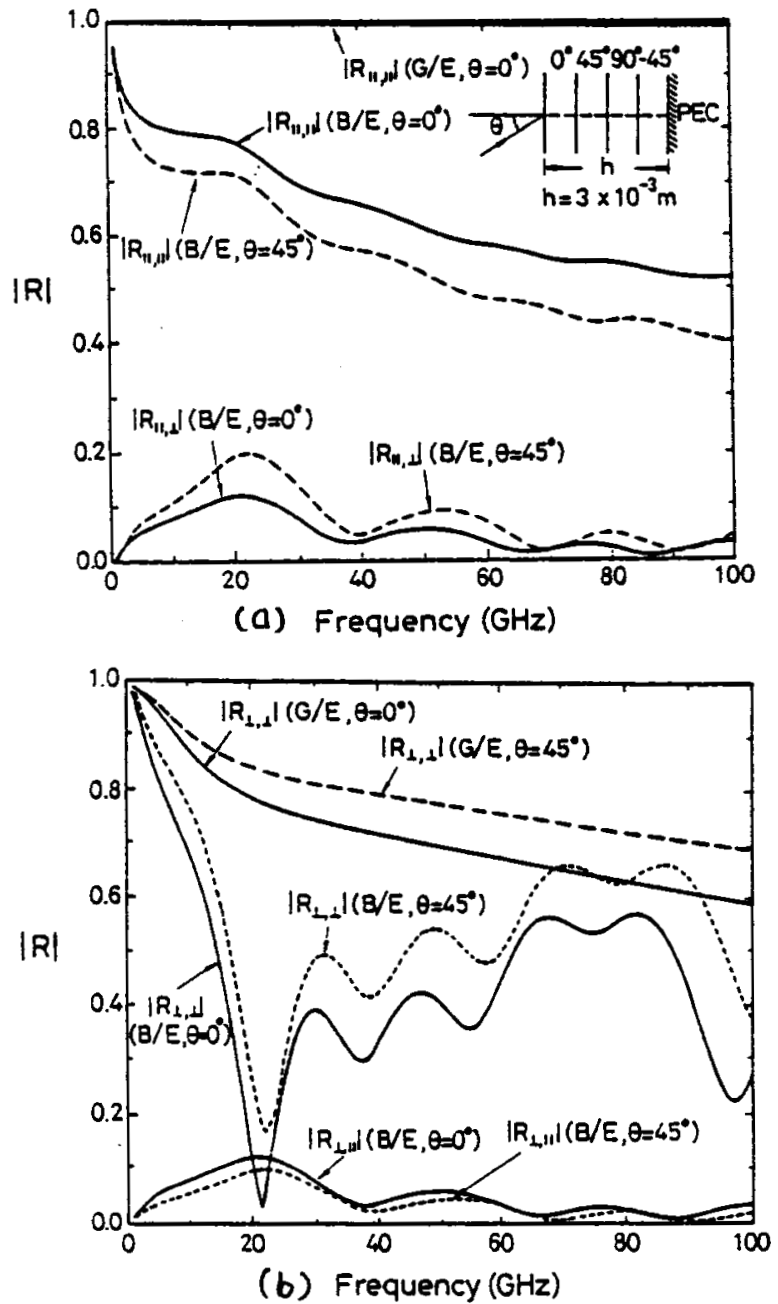

Fig.3. Time-domain response of laminated composites with Gaussian pulses ( $\tau=$ $\left.5 \times 10^{-12} s, t_{0}=5 \times 10^{-11} s\right)$ incident normally.

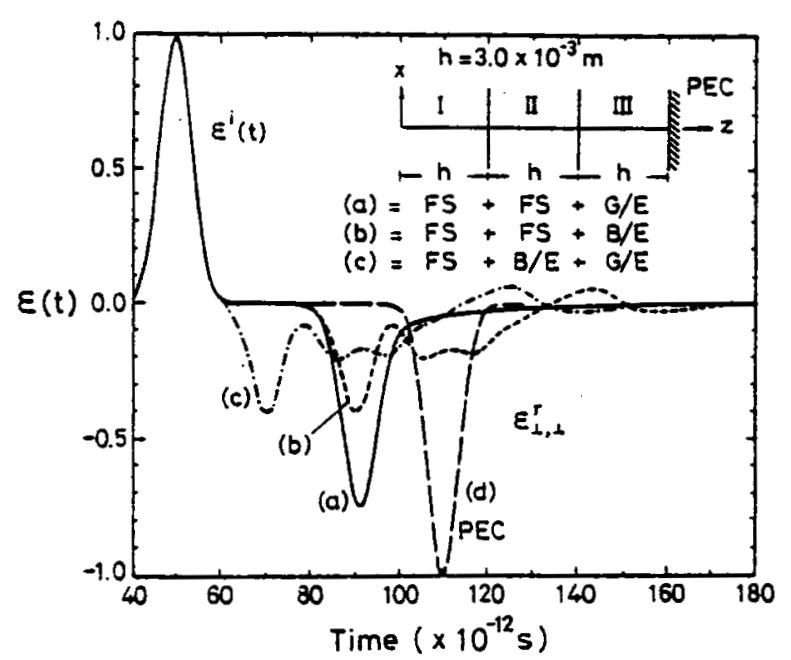

Table 1. Electrical parameters of laminated composites

\begin{tabular}{|c|c|c|c|}
\hline & $\mathrm{G} / \mathrm{E}$ & $\mathrm{B} / \mathrm{E}$ & $\mathrm{K} / \mathrm{E}$ \\
\hline$\sigma_{z^{\prime}}$ & $4.0 \times 10^{4}$ & 30 & $1.0 \times 10^{-9}$ \\
\hline$\sigma_{y^{\prime}}$ & 100 & $2.0 \times 10^{-8}$ & $1.0 \times 10^{-9}$ \\
\hline$\sigma_{z^{\prime}}$ & 100 & $2.0 \times 10^{-8}$ & $1.0 \times 10^{-9}$ \\
\hline$\epsilon_{z^{\prime}}$ & 5.0 & 5.6 & 3.6 \\
\hline$\epsilon_{y^{\prime}}$ & 5.0 & 5.6 & 3.6 \\
\hline$\epsilon_{z^{\prime}}$ & 5.0 & 5.6 & 3.6 \\
\hline
\end{tabular}

